\title{
New Types of Doubly Periodic Standing Wave Solutions for the Coupled Higgs Field Equation
}

\author{
Gui-qiong Xu \\ Department of Information Management, College of Management, Shanghai University, Shanghai 200444, China \\ Correspondence should be addressed to Gui-qiong Xu; xugq@staff.shu.edu.cn
}

Received 9 January 2014; Accepted 12 February 2014; Published 19 March 2014

Academic Editor: Weiguo Rui

Copyright (C) 2014 Gui-qiong Xu. This is an open access article distributed under the Creative Commons Attribution License, which permits unrestricted use, distribution, and reproduction in any medium, provided the original work is properly cited.

Based on the Hirota bilinear method and theta function identities, we obtain a new type of doubly periodic standing wave solutions for a coupled Higgs field equation. The Jacobi elliptic function expression and long wave limits of the periodic solutions are also presented. By selecting appropriate parameter values, we analyze the interaction properties of periodic-periodic waves and periodicsolitary waves by some figures.

\section{Introduction}

As is well known, the investigation of the explicit exact solutions to nonlinear evolution equations (NLEEs) plays an important role in the study of nonlinear physical phenomena $[1,2]$. In the past decades, much effort has been spent on the construction of various forms of periodic wave solutions for NLEEs, and many powerful methods have been developed such as the algebrogeometrical approach $[3,4]$, nonlinearization approach of Lax pairs $[5,6]$, Weierstrass elliptic function expansion method $[7,8]$, Jacobi elliptic function expansion method [9-11], and subsidiary ordinary differential equation methods [12-18].

In this paper, we will focus on a coupled Higgs field equation with important physical interests [19],

$$
\begin{gathered}
u_{t t}-u_{x x}-\beta u+\delta|u|^{2} u-2 u v=0, \\
v_{t t}+v_{x x}-\delta\left(|u|^{2}\right)_{x x}=0,
\end{gathered}
$$

which describes a system of conserved scalar nucleons interacting with neutral scalar mesons in particle physics. Here $\beta$ and $\delta$ are constants, and the function $v=v(x, t)$ represents a real scalar meson field and $u=u(x, t)$ a complex scalar nucleon field. Equation (1) is related to some nonlinear models with physical interests. Equation (1) is the coupled nonlinear Klein-Gordon equations for $\beta<0$ and $\delta<0$ and the Higgs equations for $\beta>0$ and $\delta>0$. Much attention has been paid to investigate exact explicit solutions and integrable properties of (1). The symmetry reductions, the homoclinic orbits, $N$-soliton solutions, rogue wave solutions, Jacobi periodic solutions, and other types of travelling wave solutions have been presented [19-24].

The Hirota bilinear method is a powerful tool for constructing various exact solutions for NLEEs, which include soliton, negaton, rogue waves, rational solutions, and quasiperiodic solutions [25-35]. Recently, by means of Hirota bilinear method and theta function identities [36-38], Fan et al. obtained a class of doubly periodic standing wave solutions of (1) [39], which was expressed as rational functions of elliptic/theta functions of different moduli. A significant portion of these solutions represents travelling wave, that is, those which will remain steady in an appropriate frame of reference. Physically, the envelope of these oscillations is bounded by a pattern periodic in both time and space. The focus of this work is to investigate new types of doubly periodic standing wave solutions for (1).

This paper is organized as follows. In Section 2, we briefly illustrate some properties of theta functions and Jacobi elliptic functions. In Section 3, we construct a new kind of doubly periodic wave solutions for the coupled Higgs field equation. In Section 4, for the obtained periodic solution, we derive its Jacobi elliptic function representation and analyze interaction properties by some figures. Some conclusions are given in Section 5. 


\section{The Theta and Jacobi Elliptic Functions}

The main tools used in this paper are Hirota operators and theta function formulas, which will be discussed here, to fix the notations and make our presentation self-contained. More formulas for the theta functions can be found in [36, 37].

The Riemann theta functions of genus one $\vartheta_{n}(x, \tau)(n=$ $1-4$ ), the parameter $q$ (the nome), and $\tau$ (pure imaginary) are defined by [40]

$$
\begin{aligned}
\vartheta_{1}(x, \tau) & =2 \sum_{n=0}^{\infty}(-1)^{n} q^{(n+1 / 2)^{2}} \sin (2 n+1) x, \\
\vartheta_{2}(x, \tau) & =2 \sum_{n=0}^{\infty} q^{(n+1 / 2)^{2}} \cos (2 n+1) x \\
\vartheta_{3}(x, \tau) & =1+2 \sum_{n=1}^{\infty} q^{n^{2}} \cos 2 n x \\
\vartheta_{4}(x, \tau) & =1+2 \sum_{n=1}^{\infty}(-1)^{n} q^{n^{2}} \cos 2 n x \\
0<q<1, \quad q & =\exp (i \pi \tau), \quad q=\exp \left(-\frac{\pi K^{\prime}}{K}\right) .
\end{aligned}
$$

Here, $K, K^{\prime}$ are the complete elliptic integrals of the first kind:

$$
\begin{aligned}
K & =\int_{0}^{\pi / 2}\left(1-k^{2} \sin ^{2} \xi\right)^{-1 / 2} d \xi \\
K_{1} & =\int_{0}^{\pi / 2}\left(1-k_{1}^{2} \sin ^{2} \xi\right)^{-1 / 2} d \xi
\end{aligned}
$$

$\vartheta_{1}$ is an odd function while the other three are even functions. The zeros of $\vartheta_{1}, \vartheta_{2}, \vartheta_{3}$, and $\vartheta_{4}$ are at $M \pi+N \pi \tau,(M+(1 / 2)) \pi+$ $N \pi \tau,(M+(1 / 2)) \pi+(N+(1 / 2)) \pi \tau$, and $M \pi+(N+(1 / 2)) \pi \tau$, respectively, and $M$ and $N$ are integers. Since $\vartheta_{1}, \vartheta_{2}$, and $\left(\vartheta_{3}\right.$, $\vartheta_{4}$ ) are related by a phase shift of $\pi / 2$, there are roughly two groups of theta functions.

There exists a large class of bilinear identities involving products of theta functions, some of which are listed here:

$$
\begin{aligned}
& D_{x}^{2} \vartheta_{1}(x, \tau) \cdot \vartheta_{2}(x, \tau)=\left(b_{2}+\vartheta_{2}^{4}(0, \tau)\right) \vartheta_{1}(x, \tau) \vartheta_{2}(x, \tau) \\
& D_{x}^{2} \vartheta_{1}(x, \tau) \cdot \vartheta_{4}(x, \tau)=\left(b_{2}-\vartheta_{4}^{4}(0, \tau)\right) \vartheta_{1}(x, \tau) \vartheta_{4}(x, \tau) \\
& D_{x}^{2} \vartheta_{2}(x, \tau) \cdot \vartheta_{3}(x, \tau)=\left(b_{2}-\vartheta_{4}^{4}(0, \tau)\right) \vartheta_{2}(x, \tau) \vartheta_{3}(x, \tau) \\
& D_{x}^{2} \vartheta_{1}(x, \tau) \cdot \vartheta_{3}(x, \tau)=b_{3} \vartheta_{1}(x, \tau) \vartheta_{3}(x, \tau) \\
& D_{x}^{2} \vartheta_{2}(x, \tau) \cdot \vartheta_{4}(x, \tau)=b_{3} \vartheta_{2}(x, \tau) \vartheta_{4}(x, \tau) \\
& D_{x}^{2} \vartheta_{2}(x, \tau) \cdot \vartheta_{2}(x, \tau)=b_{2} \vartheta_{2}^{2}(x, \tau)-b_{1} \vartheta_{4}^{2}(x, \tau) \\
& D_{x}^{2} \vartheta_{4}(x, \tau) \cdot \vartheta_{4}(x, \tau)=b_{1} \vartheta_{2}^{2}(x, \tau)+b_{2} \vartheta_{4}^{2}(x, \tau)
\end{aligned}
$$

where for simplicity we have used the notations

$$
\begin{gathered}
b_{1}=2 \vartheta_{2}^{2}(0, \tau) \vartheta_{4}^{2}(0, \tau), \quad b_{2}=2 \frac{\vartheta_{3}^{\prime \prime}(0, \tau)}{\vartheta_{3}(0, \tau)}, \\
b_{3}=\frac{\vartheta_{2}^{\prime \prime}(0, \tau)}{\vartheta_{2}(0, \tau)}+\frac{\vartheta_{4}^{\prime \prime}(0, \tau)}{\vartheta_{4}(0, \tau)}
\end{gathered}
$$

and the formulas (4) can be derived from product identities of theta functions; the details can be found in $[36,37]$.

There are close connections between theta functions and elliptic functions as follows:

$$
\begin{aligned}
& \frac{\vartheta_{1}(\gamma x, \tau)}{\vartheta_{4}(\gamma x, \tau)}=k^{1 / 2} \operatorname{sn}(\lambda x, k) \equiv S, \\
& \frac{\vartheta_{2}(\gamma x, \tau)}{\vartheta_{4}(\gamma x, \tau)}=k^{1 / 2}\left(1-k^{2}\right)^{-1 / 4} \operatorname{cn}(\lambda x, k) \equiv C, \\
& \frac{\vartheta_{3}(\gamma x, \tau)}{\vartheta_{4}(\gamma x, \tau)}=\left(1-k^{2}\right)^{-1 / 4} \operatorname{dn}(\lambda x, k) \equiv D,
\end{aligned}
$$

where $\lambda=\gamma \vartheta_{3}^{2}(0, \tau)$ and $k=\vartheta_{2}^{2}(0, \tau) / \vartheta_{3}^{2}(0, \tau)$,

$$
\begin{aligned}
& \frac{\vartheta_{1}\left(\omega t, \tau_{1}\right)}{\vartheta_{4}\left(\omega t, \tau_{1}\right)}=k_{1}^{1 / 2} \operatorname{sn}\left(\rho t, k_{1}\right) \equiv S_{1}, \\
& \frac{\vartheta_{2}\left(\omega t, \tau_{1}\right)}{\vartheta_{4}\left(\omega t, \tau_{1}\right)}=k_{1}^{1 / 2}\left(1-k_{1}^{2}\right)^{-1 / 4} \operatorname{cn}\left(\rho t, k_{1}\right) \equiv C_{1}, \\
& \frac{\vartheta_{3}\left(\omega t, \tau_{1}\right)}{\vartheta_{4}\left(\omega t, \tau_{1}\right)}=\left(1-k_{1}^{2}\right)^{-1 / 4} \mathrm{dn}\left(\rho t, k_{1}\right) \equiv D_{1},
\end{aligned}
$$

where $\rho=\omega \vartheta_{3}^{2}\left(0, \tau_{1}\right)$ and $k_{1}=\vartheta_{2}^{2}\left(0, \tau_{1}\right) / \vartheta_{3}^{2}\left(0, \tau_{1}\right)$. It is clearly shown that arguments of the theta and elliptic functions are related by a scale factor.

\section{A New Class of Doubly Periodic Wave Solutions}

In this section, we construct a new class of doubly periodic wave solutions by Hirota bilinear method [2]. For (1), substituting the following transformation

$$
u(x, t)=\frac{g}{f}, \quad v(x, t)=v_{0}+2(\ln f)_{x x}
$$

into (1) and integrating with respect to $x$ yield the bilinear forms

$$
\begin{gathered}
\left(D_{t}^{2}+D_{x}^{2}-\Lambda\right) f \cdot f=\delta g \cdot g^{\star}, \\
{\left[D_{t}^{2}-D_{x}^{2}-\left(\beta+2 v_{0}+\Lambda\right)\right] g \cdot f=0,}
\end{gathered}
$$

where $v_{0}$ is a constant, $\Lambda=\Lambda(t)$ is an integration constant, and $D$ is the well-known Hirota bilinear operator. Equation (10) is slightly different from the results given in [39] by adding one integration constant term.

The crucial step to derive doubly periodic wave solutions is to suppose $f$ and $g$ in (9) and (10) as suitable combination 
of different theta functions. To obtain new doubly periodic wave solutions, we make a new ansatz,

$$
\begin{gathered}
g=\eta\left[\vartheta_{3}\left(\omega t, \tau_{1}\right) \vartheta_{2}(\gamma x, \tau)\right. \\
\left.+i \vartheta_{1}\left(\omega t, \tau_{1}\right) \vartheta_{4}(\gamma x, \tau)\right] \exp (i p t), \\
f=\vartheta_{4}\left(\omega t, \tau_{1}\right) \vartheta_{4}(\gamma x, \tau)+\vartheta_{2}\left(\omega t, \tau_{1}\right) \vartheta_{2}(\gamma x, \tau),
\end{gathered}
$$

where the parameters $\omega, \gamma, \eta$, and $p$ are constants to be determined and the period $\tau$ in the spatial direction and the period $\tau_{1}$ in the temporal direction are purely imaginary constants.

Inserting the ansatz (11) into (9), together with the theta function identities given in Section 2, we set the coefficients of the terms $\vartheta_{2}^{2}(\gamma x, \tau), \vartheta_{4}^{2}(\gamma x, \tau)$, and $\vartheta_{2}(\gamma x, \tau) \vartheta_{4}(\gamma x, \tau)$ to be zero and get

$$
\begin{gathered}
\left(\gamma^{2} b_{2}+\omega^{2} \widetilde{b}_{2}-\Lambda-\delta \eta^{2} \widetilde{b}_{4}\right) \vartheta_{2}^{2}\left(\omega t, \tau_{1}\right) \\
+\left(\gamma^{2} b_{1}-\omega^{2} \widetilde{b}_{1}-\delta \eta^{2} \widetilde{b}_{5}\right) \vartheta_{4}^{2}\left(\omega t, \tau_{1}\right)=0, \\
\left(\gamma^{2} b_{1}-\omega^{2} \widetilde{b}_{1}-\delta \eta^{2} \widetilde{b}_{5}\right) \vartheta_{2}^{2}\left(\omega t, \tau_{1}\right) \\
-\left(\gamma^{2} b_{2}+\omega^{2} \widetilde{b}_{2}-\Lambda-\delta \eta^{2} \widetilde{b}_{4}\right) \vartheta_{4}^{2}\left(\omega t, \tau_{1}\right)=0, \\
\left(\gamma^{2} b_{3}+\omega^{2} \widetilde{b}_{3}-\Lambda\right) \vartheta_{2}\left(\omega t, \tau_{1}\right) \vartheta_{4}\left(\omega t, \tau_{1}\right)=0,
\end{gathered}
$$

where the theta constants $\widetilde{b}_{j}(j=1, \ldots, 5)$ are defined by

$$
\begin{gathered}
\tilde{b}_{1}=2 \vartheta_{2}^{2}\left(0, \tau_{1}\right) \vartheta_{4}^{2}\left(0, \tau_{1}\right), \quad \tilde{b}_{2}=\frac{2 \vartheta_{3}^{\prime \prime}\left(0, \tau_{1}\right)}{\vartheta_{3}\left(0, \tau_{1}\right)} \\
\tilde{b}_{3}=\frac{\vartheta_{2}^{\prime \prime}\left(0, \tau_{1}\right)}{\vartheta_{2}\left(0, \tau_{1}\right)}+\frac{\vartheta_{4}^{\prime \prime}\left(0, \tau_{1}\right)}{\vartheta_{4}\left(0, \tau_{1}\right)} \\
\tilde{b}_{4}=\frac{\vartheta_{2}^{2}\left(0, \tau_{1}\right)}{\vartheta_{3}^{2}\left(0, \tau_{1}\right)}, \quad \tilde{b}_{5}=\frac{\vartheta_{4}^{2}\left(0, \tau_{1}\right)}{\vartheta_{3}^{2}\left(0, \tau_{1}\right)}
\end{gathered}
$$

Due to the linear independence of theta functions, we set the coefficients of $\vartheta_{2}^{2}\left(\omega t, \tau_{1}\right), \vartheta_{4}^{2}\left(\omega t, \tau_{1}\right)$, and $\vartheta_{2}\left(\omega t, \tau_{1}\right) \vartheta_{4}\left(\omega t, \tau_{1}\right)$ in $(12)$ to be zero and obtain

$$
\begin{gathered}
\gamma^{2} b_{1}-\omega^{2} \widetilde{b}_{1}-\delta \eta^{2} \widetilde{b}_{5}=0 \\
\gamma^{2} b_{2}+\omega^{2} \widetilde{b}_{2}-\Lambda-\delta \eta^{2} \widetilde{b}_{4}=0 \\
\gamma^{2} b_{3}+\omega^{2} \widetilde{b}_{3}-\Lambda=0
\end{gathered}
$$

Similarly, substituting the ansatz (11) into (10) and applying identities for Hirota derivatives of theta functions yield

$$
\begin{aligned}
& \left(\omega^{2}\left(\widetilde{b}_{2}-\vartheta_{4}^{4}\left(0, \tau_{1}\right)\right)-\gamma^{2} b_{2}-\left(\beta+2 v_{0}+\Lambda+p^{2}\right)\right) \\
& \times \vartheta_{2}\left(\omega t, \tau_{1}\right) \vartheta_{3}\left(\omega t, \tau_{1}\right) \vartheta_{2}^{2}(\gamma x, \tau) \\
& +\left(\gamma^{2} b_{1}-2 p \omega \vartheta_{4}^{2}\left(0, \tau_{1}\right)\right) \vartheta_{2}\left(\omega t, \tau_{1}\right) \vartheta_{3}\left(\omega t, \tau_{1}\right) \vartheta_{4}^{2}(\gamma x, \tau) \\
& +\left(\omega^{2}\left(\widetilde{b}_{2}+\vartheta_{2}^{4}\left(0, \tau_{1}\right)\right)-\gamma^{2} b_{3}-2 p \omega \vartheta_{2}^{2}\left(0, \tau_{1}\right)\right. \\
& \left.\quad-\left(\beta+2 v_{0}+\Lambda+p^{2}\right)\right) \vartheta_{3} \\
& \times\left(\omega t, \tau_{1}\right) \vartheta_{4}\left(\omega t, \tau_{1}\right) \vartheta_{2}(\gamma x, \tau) \vartheta_{4}(\gamma x, \tau) \\
& +i\left[-\left(\gamma^{2} b_{1}-2 p \omega \vartheta_{4}^{2}\left(0, \tau_{1}\right)\right) \vartheta_{1}\left(\omega t, \tau_{1}\right) \vartheta_{4}\left(\omega t, \tau_{1}\right) \vartheta_{2}^{2}(\gamma x, \tau)\right. \\
& +\left(\omega^{2}\left(\widetilde{b}_{2}+\vartheta_{2}^{4}\left(0, \tau_{1}\right)\right)-\gamma^{2} b_{3}-2 p \omega \vartheta_{2}^{2}\left(0, \tau_{1}\right)\right. \\
& \left.\quad-\left(\beta+2 v_{0}+\Lambda+p^{2}\right)\right) \\
& \quad \times \vartheta_{1}\left(\omega t, \tau_{1}\right) \vartheta_{2}\left(\omega t, \tau_{1}\right) \vartheta_{2}(\gamma x, \tau) \vartheta_{4}(\gamma x, \tau) \\
& +\left(\omega^{2}\left(\widetilde{b}_{2}-\vartheta_{4}^{4}\left(0, \tau_{1}\right)\right)-\gamma^{2} b_{2}-\left(\beta+2 v_{0}+\Lambda+p^{2}\right)\right) \\
& \left.\times \vartheta_{1}\left(\omega t, \tau_{1}\right) \vartheta_{4}\left(\omega t, \tau_{1}\right) \vartheta_{4}^{2}(\gamma x, \tau)\right]=0 .
\end{aligned}
$$

For the real part and the imaginary part of (15), setting the coefficients of the terms $\vartheta_{2}^{2}(\gamma x, \tau), \vartheta_{4}^{2}(\gamma x, \tau)$, and $\vartheta_{2}(\gamma x, \tau) \vartheta_{4}(\gamma x, \tau)$ to be zero yields an algebraic system as follows:

$$
\begin{gathered}
\gamma^{2} b_{1}-2 p \omega \vartheta_{4}^{2}\left(0, \tau_{1}\right)=0, \\
\omega^{2}\left(\widetilde{b}_{2}-\vartheta_{4}^{4}\left(0, \tau_{1}\right)\right)-\gamma^{2} b_{2}-\left(\beta+2 v_{0}+\Lambda+p^{2}\right)=0, \\
\omega^{2}\left(\widetilde{b}_{2}+\vartheta_{2}^{4}\left(0, \tau_{1}\right)\right)-\gamma^{2} b_{3}-2 p \omega \vartheta_{2}^{2}\left(0, \tau_{1}\right) \\
-\left(\beta+2 v_{0}+\Lambda+p^{2}\right)=0 .
\end{gathered}
$$

Solving the algebraic system given by (14) and (16) with respect to the variables $v_{0}, \eta, p, \gamma, \omega, \tau, \tau_{1}$, and $\Lambda$, one obtains a set of nontrivial solutions:

$$
\begin{gathered}
\omega^{2}=\left(\frac{b_{1} \widetilde{b}_{4}-\widetilde{b}_{5}\left(b_{2}-b_{3}\right)}{\widetilde{b}_{1} \widetilde{b}_{4}+\left(\widetilde{b}_{2}-\widetilde{b}_{3}\right) \widetilde{b}_{5}}\right) \gamma^{2}, \quad p=\frac{b_{1} \gamma^{2}}{2 \omega \vartheta_{4}^{2}\left(0, \tau_{1}\right)}, \\
\Lambda=b_{3} \gamma^{2}+\widetilde{b}_{3} \omega^{2}, \quad \eta^{2}=\frac{\gamma^{2}\left(b_{2}-b_{3}\right)+\omega^{2}\left(\widetilde{b}_{2}-\widetilde{b}_{3}\right)}{\delta \widetilde{b}_{4}}, \\
v_{0}=\frac{\omega^{2}\left(\widetilde{b}_{2}-\vartheta_{4}^{4}\left(0, \tau_{1}\right)\right)}{2}-\frac{b_{2} \gamma^{2}+\beta+\Lambda+p^{2}}{2},
\end{gathered}
$$

where the parameters $\gamma, \tau$, and $\tau_{1}$ are arbitrary constants. 

of (1):

Therefore, we obtain a new doubly periodic wave solution

$$
\begin{aligned}
& u(x, t) \\
& =\frac{\eta\left[\vartheta_{3}\left(\omega t, \tau_{1}\right) \vartheta_{2}(\gamma x, \tau)+i \vartheta_{1}\left(\omega t, \tau_{1}\right) \vartheta_{4}(\gamma x, \tau)\right] \exp (i p t)}{\vartheta_{4}\left(\omega t, \tau_{1}\right) \vartheta_{4}(\gamma x, \tau)+\vartheta_{2}\left(\omega t, \tau_{1}\right) \vartheta_{2}(\gamma x, \tau)}, \\
& v(x, t) \\
& =v_{0}+2\left(\operatorname { l n } \left[\vartheta_{4}\left(\omega t, \tau_{1}\right) \vartheta_{4}(\gamma x, \tau)\right.\right. \\
& \left.\left.\quad+\vartheta_{2}\left(\omega t, \tau_{1}\right) \vartheta_{2}(\gamma x, \tau)\right]\right)_{x x},
\end{aligned}
$$

where $\gamma, \tau$, and $\tau_{1}$ are arbitrary constants, and the parameters $\omega, p, \eta, v_{0}$, and $\Lambda$ are given by (17). To the author's knowledge, the solution (18) is firstly reported here.

In fact, the coupled Higgs field equation (1) admits abundant families of doubly periodic wave solutions. For example, we can suppose the solutions of the bilinear equations (9) and (10) as

$$
\begin{gathered}
g=\eta\left[\vartheta_{1}\left(\omega t, \tau_{1}\right) \vartheta_{3}(\gamma x, \tau)\right. \\
\left.+i \vartheta_{2}\left(\omega t, \tau_{1}\right) \vartheta_{4}(\gamma x, \tau)\right] \exp (i p t), \\
f=\vartheta_{4}\left(\omega t, \tau_{1}\right) \vartheta_{4}(\gamma x, \tau)+\vartheta_{3}\left(\omega t, \tau_{1}\right) \vartheta_{3}(\gamma x, \tau),
\end{gathered}
$$

or

$$
\begin{aligned}
& g=\eta {\left[\vartheta_{3}\left(\omega t, \tau_{1}\right) \vartheta_{4}(\gamma x, \tau)\right.} \\
&\left.+i \vartheta_{1}\left(\omega t, \tau_{1}\right) \vartheta_{2}(\gamma x, \tau)\right] \exp (i p t), \\
& f=\vartheta_{4}\left(\omega t, \tau_{1}\right) \vartheta_{2}(\gamma x, \tau) \\
&+\vartheta_{2}\left(\omega t, \tau_{1}\right) \vartheta_{4}(\gamma x, \tau)
\end{aligned}
$$

and so on. For the sake of simplicity, the tedious computations are omitted here. It is noted that these two types of periodic solutions have also two independent periods in the spatial and temporal directions.

\section{Jacobi Elliptic Function Expressions and Long Wave Limit}

In order to analyze the periodic property by some figures, we may first convert solution (18) into Jacobi elliptic function expressions. Together with (6) and (7), solution (18) can be expressed as rational forms of Jacobi elliptic functions:

$$
\begin{gathered}
u(x, t) \\
= \pm \sqrt{\frac{\lambda^{2}\left(1-2 k^{2}\right)+\rho^{2}\left(1-2 k_{1}^{2}\right)}{\delta k_{1}}} \frac{D_{1} C+i S_{1}}{1+C_{1} C} \exp (i p t) \\
v(x, t)=v_{0}+2 \lambda^{2}\left(1-k^{2}-\frac{E}{K}\right) \\
+\frac{2 \lambda^{2}\left[k \sqrt{1-k^{2}}\left(C^{2}-C_{1}^{2}\right)+\left(2 k^{2}-1\right) C C_{1}\right]}{\left(1+C_{1} C\right)^{2}},
\end{gathered}
$$

where $\lambda$ is an arbitrary constant, and the parameters $\rho, p, \Lambda$, and $v_{0}$ are given by

$$
\begin{aligned}
\rho^{2} & =\lambda^{2}\left[2 k^{2}-1+2 k k_{1}\left(1-k^{2}\right)^{1 / 2}\left(1-k_{1}^{2}\right)^{-1 / 2}\right], \\
p & =\frac{\lambda^{2}}{\rho} k\left(1-k^{2}\right)^{1 / 2}\left(1-k_{1}^{2}\right)^{-1 / 2}, \\
\Lambda & =\lambda^{2}\left(1-\frac{2 E}{K}\right)+\rho^{2}\left(1-\frac{2 E_{1}}{K_{1}}\right), \\
v_{0} & =\rho^{2}\left(\frac{\left(1-k_{1}^{2}\right)}{2}-\frac{E_{1}}{K_{1}}\right)-\lambda^{2}\left(1-k^{2}-\frac{E}{K}\right)-\frac{\beta+p^{2}+\Lambda}{2},
\end{aligned}
$$

which indicates that the period $k$ in the spatial direction and the period $k_{1}$ in the temporal direction are related by

$$
2 k^{2}-1+2 k k_{1}\left(1-k^{2}\right)^{1 / 2}\left(1-k_{1}^{2}\right)^{-1 / 2}>0 .
$$

In (21) and (22), the complete elliptic integrals $E$ and $E_{1}$ are defined by

$$
E=\int_{0}^{\pi / 2} \sqrt{1-k^{2} \sin ^{2} \xi} d \xi, \quad E_{1}=\int_{0}^{\pi / 2} \sqrt{1-k_{1}^{2} \sin ^{2} \xi} d \xi
$$

From (21), it is easy to check that

$$
\begin{array}{cl}
u(x, t)=u\left(x+\frac{4 K}{\lambda}, t\right), & |u(x, t)|=\left|u\left(x, t+\frac{4 K_{1}}{\rho}\right)\right|, \\
v(x, t)=v\left(x+\frac{4 K}{\lambda}, t\right), & v(x, t)=v\left(x, t+\frac{4 K_{1}}{\rho}\right),
\end{array}
$$

which implies that the solution $(|u|, v)$ is periodic in the $x$-direction with a period $4 K / \lambda$ and the $t$-direction with a period $4 K_{1} / \rho$.

By selecting appropriate parameter values in (21), the interactions of doubly periodic waves are shown in Figures 1 and 2. It is clearly seen that $(|u|, v)$ is periodic in the $x$-direction and the $t$-direction. For the solution in [39], the periodic waves are both bell shaped in the spatial and temporal directions. However, with regard to solution (21), the periodic waves are of different shapes in the spatial and temporal directions.

When the modulus $k \rightarrow 1,(\operatorname{sn} x, \operatorname{cn} x, \operatorname{dn} x) \rightarrow$ $(\tanh x, \operatorname{sech} x, \operatorname{sech} x)$. And if $k \rightarrow 0,(\operatorname{sn} x, \operatorname{cn} x, \operatorname{dn} x) \rightarrow$ $(\sin x, \cos x, 1)$. Therefore, the long wave limits of the periodic wave solutions can be readily obtained. A long wave limit of the solution (21) can be taken by assuming

$$
\frac{\sqrt{k}}{\left(1-k_{1}^{2}\right)^{1 / 4}}=m, \quad 0<m<1
$$




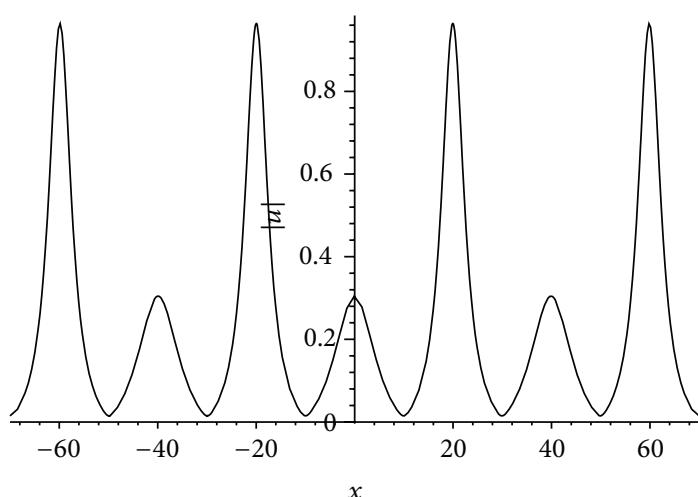

(a) In $x$-direction

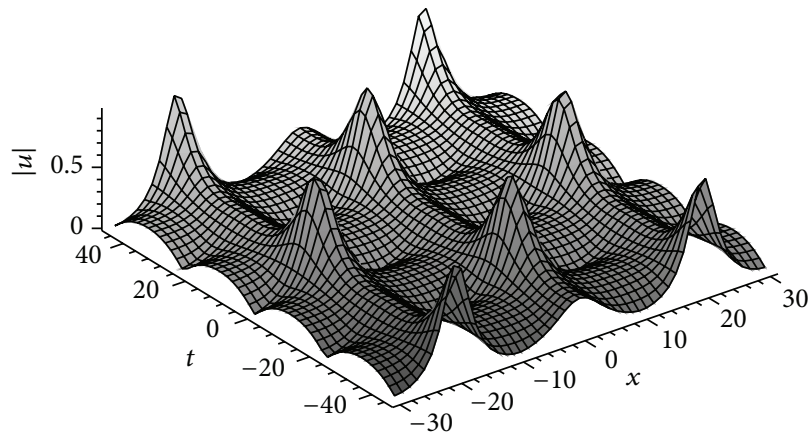

(c) Evolution plot of $|u|$

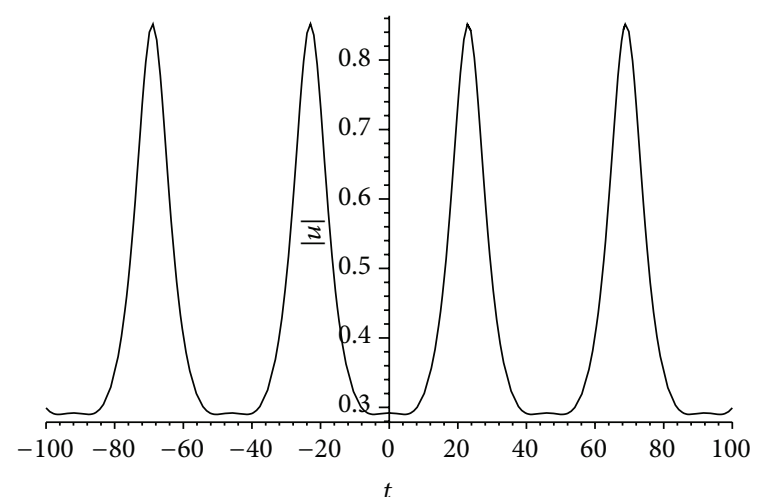

(b) In $t$-direction

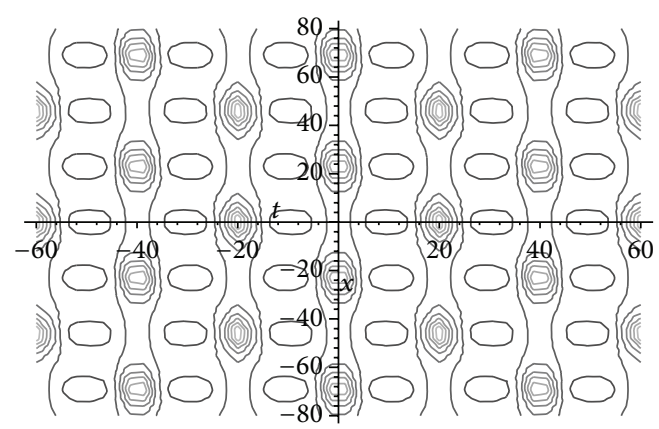

(d) Contour plot

FIGURE 1: Doubly periodic wave solution $u(x, t)$ given by (21) with $\delta=0.2, \beta=0.4, \lambda=0.2, k=0.8$, and $k_{1}=0.2$.

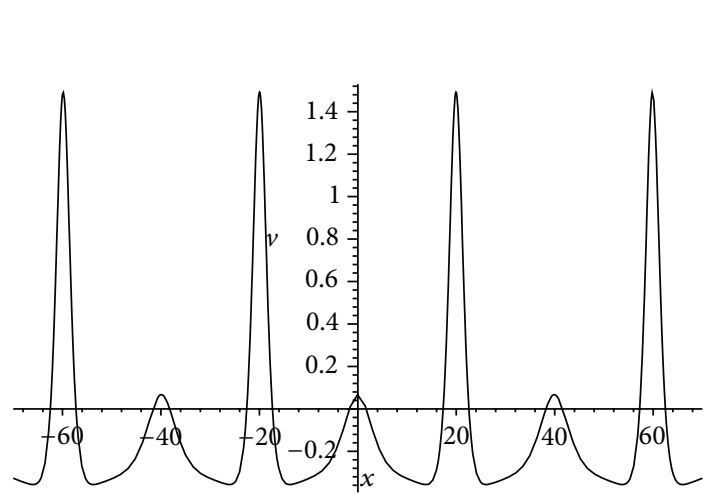

(a) In $x$-direction

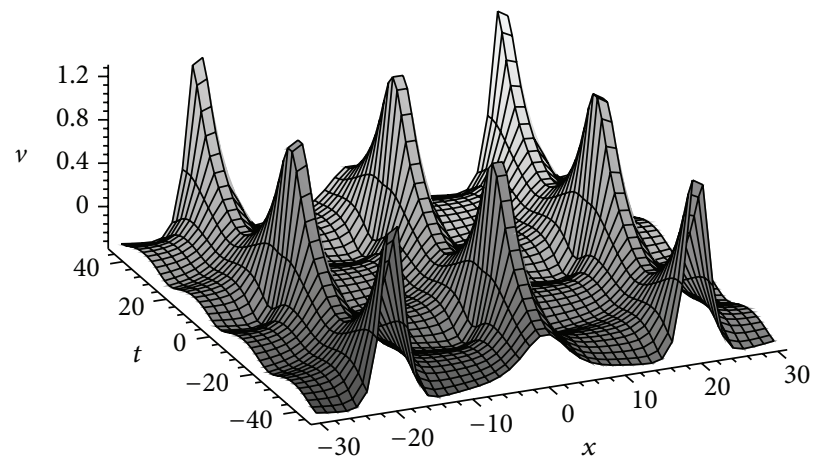

(c) Evolution plot of $v$

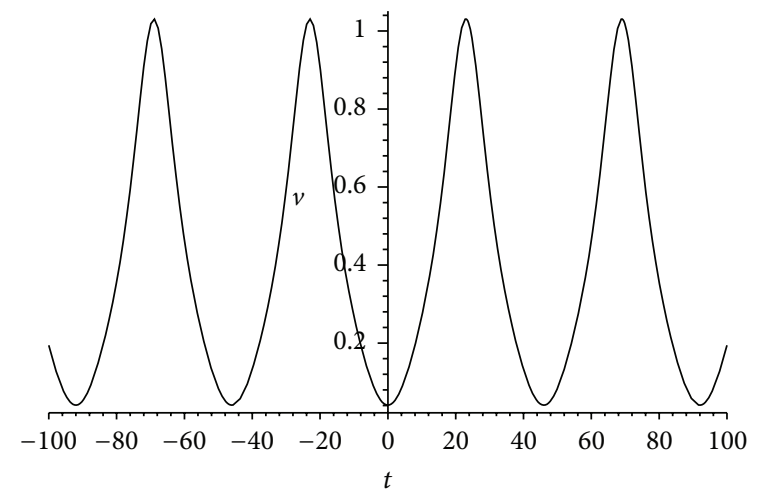

(b) In $t$-direction

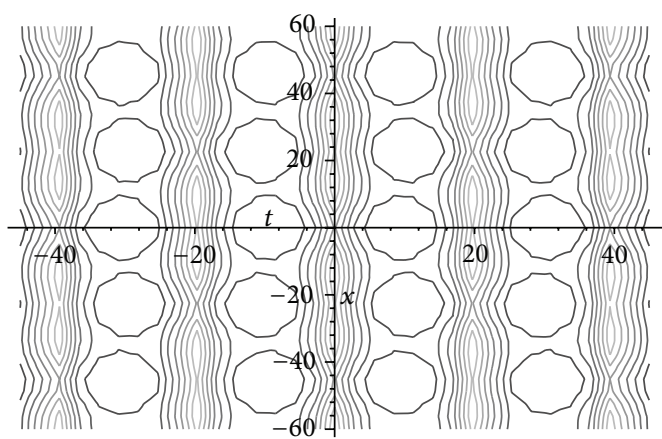

(d) Contour plot

Figure 2: Doubly periodic wave solution $v(x, t)$ given by (21) with $\delta=0.2, \beta=0.4, \lambda=0.2, k=0.8$, and $k_{1}=0.2$. 


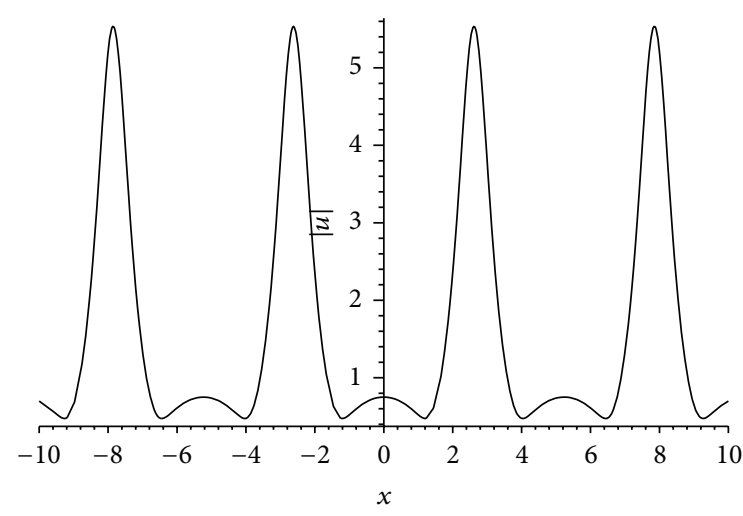

(a) In $x$-direction

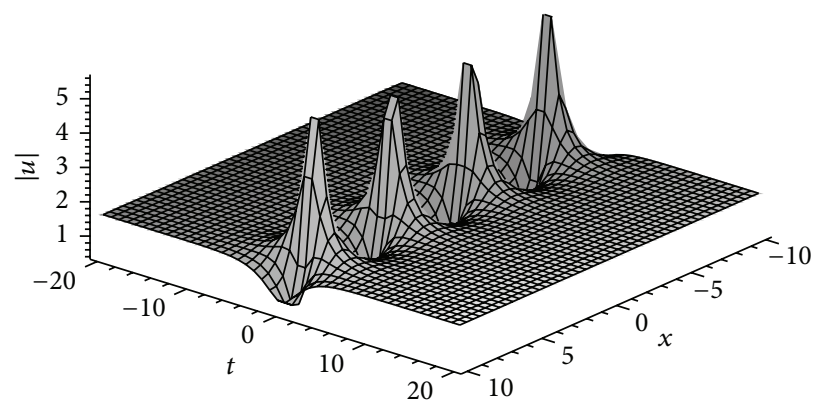

(c) Evolution plot of $|u|$

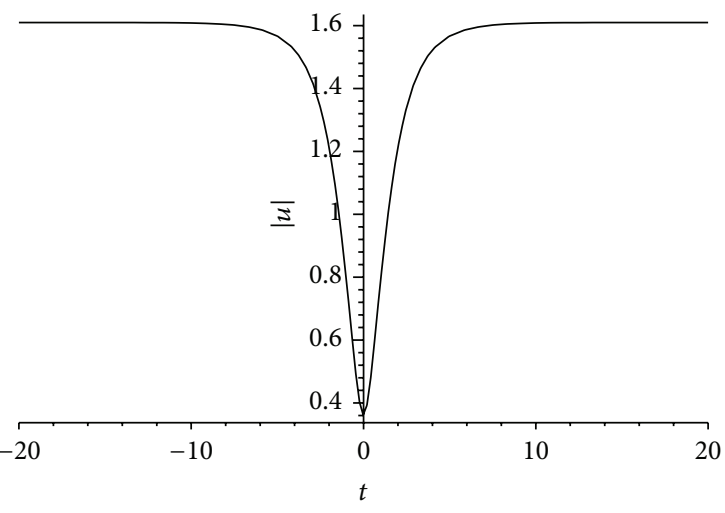

(b) In $t$-direction

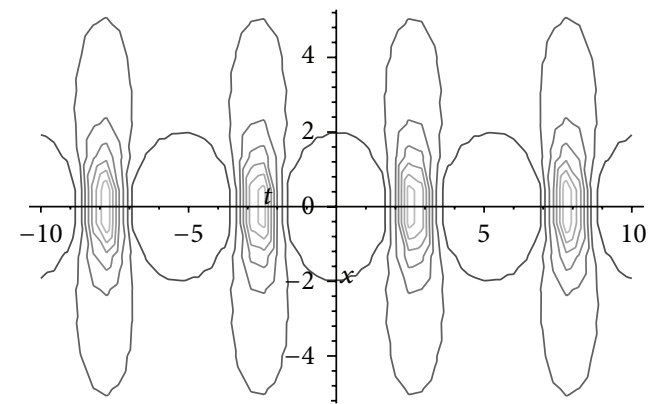

(d) Contour plot

FIGURE 3: The periodic-solitary wave solution $u(x, t)$ given by $(27)$ with $\delta=0.4, \beta=2.0, \lambda=1.2$, and $m=0.8$.

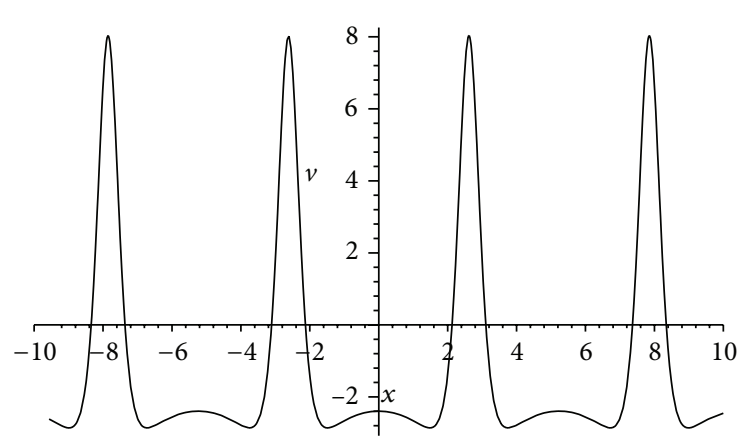

(a) In $x$-direction

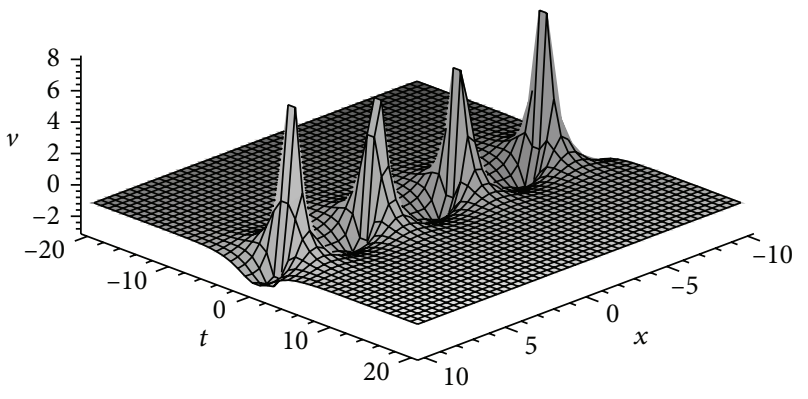

(c) Evolution plot of $v$

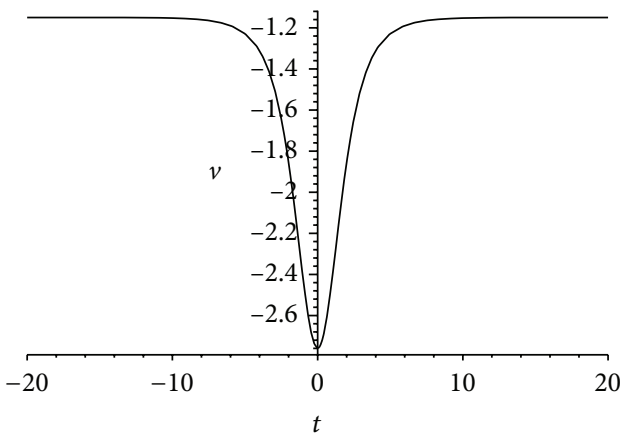

(b) In $t$-direction

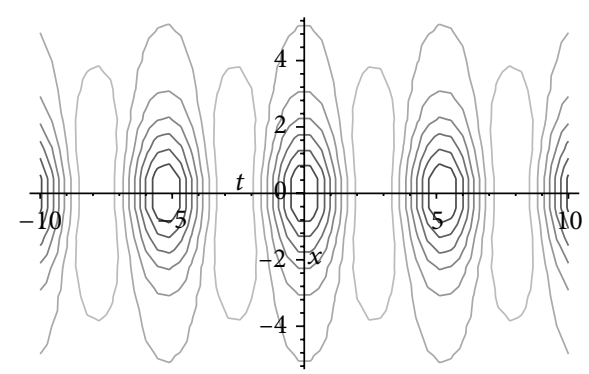

(d) Contour plot

FIGURE 4: The periodic-solitary wave solution $v(x, t)$ given by (27) with $\delta=0.4, \beta=2.0, \lambda=1.2$, and $m=0.8$. 
When the modulus $k \rightarrow 0$ and $k_{1} \rightarrow 1$, one obtains a new periodic-solitary wave solution as follows:

$$
\begin{gathered}
u=\eta\left[\frac{m \cos (\lambda x) \operatorname{sech}(\rho t)+i \tanh (\rho t)}{1+m \cos (\lambda x) \operatorname{sech}(\rho t)}\right] \exp (i p t), \\
v=-\frac{\beta}{2}-\frac{\lambda^{2}\left(5 m^{4}-6 m^{2}+2\right)}{2\left(2 m^{2}-1\right)} \\
-\frac{2 m \lambda^{2} \operatorname{sech}(\rho t)(m \operatorname{sech}(\rho t)+\cos (\lambda x))}{(1+m \cos (\lambda x) \operatorname{sech}(\rho t))^{2}}
\end{gathered}
$$

where the parameters $\rho, p$, and $\eta$ are given by

$$
\begin{gathered}
\rho=\lambda \sqrt{2 m^{2}-1}, \quad p=\frac{\lambda m^{2}}{\sqrt{2 m^{2}-1}}, \\
\eta=\lambda \sqrt{\frac{2\left(1-m^{2}\right)}{\delta}} .
\end{gathered}
$$

With proper selections of the values of $\beta, \delta, \lambda$, and $m$, the interactions of periodic solitary waves (27) are shown in Figures 3 and 4 . The solution $(|u|, v)$ displays the feature of a dark soliton in the $t$-direction; the cosine function causes periodic modulation and thus it is periodic in the $x$-direction.

With the aid of the computer algebra software Maple, the validity of the new solutions (18) and (27) are verified by putting them back into the original systems (1).

\section{Conclusions}

The combination of the Hirota bilinear method and theta function identities is demonstrated to be a powerful tool in finding periodic waves for the coupled Higgs field equation. As a result, we have derived a new kind of doubly periodic standing wave solutions for the coupled Higgs field equation, which is different from those of the known solutions reported in the literature. The interaction properties of periodicperiodic waves and periodic-solitary waves are analyzed by some figures.

The key of the combination method is that the solutions are supposed as rational expressions of elliptic functions of different moduli, which should be applicable to other nonlinear evolution equations or systems with bilinear forms in mathematical physics. The doubly periodic solutions will prove to be beneficial and instructive in modeling and understanding nonlinear phenomenon.

\section{Conflict of Interests}

The author declares that there is no conflict of interests regarding the publication of this paper.

\section{Acknowledgment}

This work was supported by the Natural Science Foundation of China under Grant no. 11201290.

\section{References}

[1] M. J. Ablowitz and P. A. Clarkson, Solitons, Nonlinear Evolution Equations and Inverse Scattering, vol. 149 of London Mathematical Society Lecture Note Series, Cambridge University Press, Cambridge, UK, 1991.

[2] R. Hirota, The Direct Method in Soliton Theory, vol. 155 of Cambridge Tracts in Mathematics, Cambridge University Press, Cambridge, UK, 2004.

[3] A. Nakamura, "A direct method of calculating periodic wave solutions to nonlinear evolution equations. I. Exact twoperiodic wave solution," Journal of the Physical Society of Japan, vol. 47, no. 5, pp. 1701-1705, 1979.

[4] F. Gesztesy and H. Holden, Soliton Equations and Their AlgebroGeometric Solutions, vol. 79 of Cambridge Studies in Advanced Mathematics, Cambridge University Press, Cambridge, UK, 2003.

[5] X. G. Geng, Y. T. Wu, and C. W. Cao, "Quasi-periodic solutions of the modified Kadomtsev-Petviashvili equation," Journal of Physics A: Mathematical and General, vol. 32, no. 20, pp. 37333742, 1999.

[6] X. G. Geng, "Algebraic-geometrical solutions of some multidimensional nonlinear evolution equations," Journal of Physics A: Mathematical and General, vol. 36, no. 9, pp. 2289-2303, 2003.

[7] J. P. Boyd, "Theta functions, Gaussian series, and spatially periodic solutions of the Korteweg-de Vries equation," Journal of Mathematical Physics, vol. 23, no. 3, pp. 375-387, 1982.

[8] A. V. Porubov, "Periodical solution to the nonlinear dissipative equation for surface waves in a convecting liquid layer," Physics Letters A, vol. 221, no. 6, pp. 391-394, 1996.

[9] S. K. Liu, Z. T. Fu, S. D. Liu, and Q. Zhao, "Jacobi elliptic function expansion method and periodic wave solutions of nonlinear wave equations," Physics Letters A, vol. 289, no. 1-2, pp. 69-74, 2001.

[10] G. Q. Xu and Z. B. Li, "Applications of Jacobi elliptic function expansion method for nonlinear differential-difference equations," Communications in Theoretical Physics, vol. 43, no. 3, pp. 385-388, 2005.

[11] G. Q. Xu, "New types of exact solutions for the fourth-order dispersive cubic-quintic nonlinear Schrödinger equation," Applied Mathematics and Computation, vol. 217, no. 12, pp. 5967-5971, 2011.

[12] S. Y. Lou and G. J. Ni, "The relations among a special type of solutions in some $(D+1)$-dimensional nonlinear equations," Journal of Mathematical Physics, vol. 30, no. 7, pp. 1614-1620, 1989.

[13] E. G. Fan, "Extended tanh-function method and its applications to nonlinear equations," Physics Letters A, vol. 277, no. 4-5, pp. $212-218,2000$

[14] S. Y. Lou, H. C. Hu, and X. Y. Tang, "Interactions among periodic waves and solitary waves of the $(N+1)$-dimensional sine-Gordon field," Physical Review E-Statistical, Nonlinear, and Soft Matter Physics, vol. 71, no. 3, Article ID 036604, 2005.

[15] G. Q. Xu, "An elliptic equation method and its applications in nonlinear evolution equations," Chaos, Solitons and Fractals, vol. 29, no. 4, pp. 942-947, 2006.

[16] W. G. Rui, B. He, and Y. Long, "The binary F-expansion method and its application for solving the $(n+1)$-dimensional sineGordon equation," Communications in Nonlinear Science and Numerical Simulation, vol. 14, no. 4, pp. 1245-1258, 2009.

[17] W. G. Rui, C. Chen, X. S. Yang, and Y. Long, "Some new solitonlike solutions and periodic wave solutions with loop or without 
loop to a generalized KdV equation," Applied Mathematics and Computation, vol. 217, no. 4, pp. 1666-1677, 2010.

[18] Y. Lei and S. Y. Lou, "Interactions among periodic waves and solitary waves of the $(2+1)$-dimensional KonopelchenkoDubrovsky equation," Chinese Physics Letters, vol. 30, no. 6, Article ID 060202, 2013.

[19] M. Tajiri, "On $N$-soliton solutions of coupled Higgs field equation," Journal of the Physical Society of Japan, vol. 52, no. 7, pp. 2277-2280, 1983.

[20] X. B. Hu, B. L. Guo, and H. W. Tam, "Homoclinic orbits for the coupled Schrödinger-Boussinesq equation and coupled higgs equation," Journal of the Physical Society of Japan, vol. 72, no. 1, pp. 189-190, 2003.

[21] Y. C. Hon and E. G. Fan, "A series of exact solutions for coupled Higgs field equation and coupled Schrödinger-Boussinesq equation," Nonlinear Analysis: Theory, Methods \& Applications, vol. 71, no. 7-8, pp. 3501-3508, 2009.

[22] G. Mu and Z. Y. Qin, "Rogue waves for the coupled SchrödingerBoussinesq equation and the coupled Higgs equation," Journal of the Physical Society of Japan, vol. 81, no. 8, Article ID 084001, 2012.

[23] A. M. Wazwaz, "Abundant soliton and periodic wave solutions for the coupled Higgs field equation, the Maccari system and the Hirota-Maccari system," Physica Scripta, vol. 85, no. 6, Article ID 065011, 2012.

[24] B. Talukdar, S. K. Ghosh, A. Saha, and D. Pal, "Solutions of the coupled Higgs field equations," Physical Review E-Statistical, Nonlinear, and Soft Matter Physics, vol. 88, no. 1, Article ID 015201, 2013.

[25] X. B. Hu and P. A. Clarkson, "Rational solutions of a differentialdifference $\mathrm{KdV}$ equation, the Toda equation and the discrete KdV equation," Journal of Physics A: Mathematical and General, vol. 28, no. 17, pp. 5009-5016, 1995.

[26] X. B. Hu and Z. N. Zhu, "A Bäcklund transformation and nonlinear superposition formula for the Belov-Chaltikian lattice," Journal of Physics A: Mathematical and General, vol. 31, no. 20, pp. 4755-4761, 1998.

[27] C. R. Gilson, X. B. Hu, W. X. Ma, and H. W. Tam, "Two integrable differential-difference equations derived from the discrete BKP equation and their related equations," Physica D: Nonlinear Phenomena, vol. 175, no. 3-4, pp. 177-184, 2003.

[28] D. J. Zhang and D. Y. Chen, "Negatons, positions, rationallike solutions and conservation laws of the Korteweg-de Vries equation with loss and non-uniformity terms," Journal of Physics A: Mathematical and General, vol. 37, no. 3, pp. 851-865, 2004.

[29] E. G. Fan and Y. C. Hon, "Quasiperiodic waves and asymptotic behavior for Bogoyavlenskii's breaking soliton equation in $(2+$ 1) dimensions," Physical Review E-Statistical, Nonlinear, and Soft Matter Physics, vol. 78, no. 3, Article ID 036607, 2008.

[30] W. X. Ma and E. G. Fan, "Linear superposition principle applying to Hirota bilinear equations," Computers \& Mathematics with Applications, vol. 61, no. 4, pp. 950-959, 2011.

[31] W. X. Ma and Z. N. Zhu, "Solving the $(3+1)$-dimensional generalized KP and BKP equations by the multiple exp-function algorithm," Applied Mathematics and Computation, vol. 218, no. 24, pp. 11871-11879, 2012.

[32] Y. Zhang, Y. Song, L. Cheng, J. Y. Ge, and W. W. Wei, "Exact solutions and Painlevé analysis of a new $(2+1)$-dimensional generalized KdV equation," Nonlinear Dynamics, vol. 68, no. 4 , pp. $445-458,2012$.
[33] Y.H. Wang and Y. Chen, "Binary Bell polynomial manipulations on the integrability of a generalized $(2+1)$-dimensional Korteweg-de Vries equation," Journal of Mathematical Analysis and Applications, vol. 400, no. 2, pp. 624-634, 2013.

[34] G. Q. Xu, "Painlevé integrability of generalized fifth-order KdV equation with variable coefficients: exact solutions and their interactions," Chinese Physics B, vol. 22, no. 5, Article ID 050203, 2013.

[35] G. Q. Xu and X. Z. Huang, "New variable separation solutions for two nonlinear evolution equations in higher dimensions," Chinese Physics Letters, vol. 30, no. 3, Article ID 130202, 2013.

[36] K. W. Chow, "A class of exact, periodic solutions of nonlinear envelope equations," Journal of Mathematical Physics, vol. 36, no. 8, pp. 4125-4137, 1995.

[37] K. W. Chow, "A class of doubly periodic waves for nonlinear evolution equations," Wave Motion, vol. 35, no. 1, pp. 71-90, 2002.

[38] J. H. Li, S. Y. Lou, and K. W. Chow, "Doubly periodic patterns of modulated hydrodynamic waves: exact solutions of the DaveyStewartson system," Acta Mechanica Sinica, vol. 27, no. 5, pp. 620-626, 2011.

[39] E. G. Fan, K. W. Chow, and J. H. Li, "On doubly periodic standing wave solutions of the coupled Higgs field equation," Studies in Applied Mathematics, vol. 128, no. 1, pp. 86-105, 2012.

[40] D. F. Lawden, Elliptic Functions and Applications, vol. 80 of Applied Mathematical Sciences, Springer-Verlag, New York, NY ,USA, 1989. 


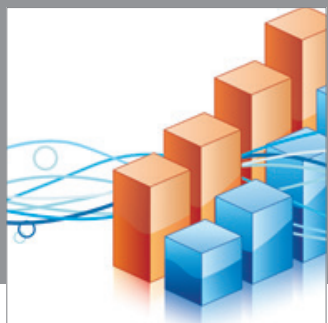

Advances in

Operations Research

mansans

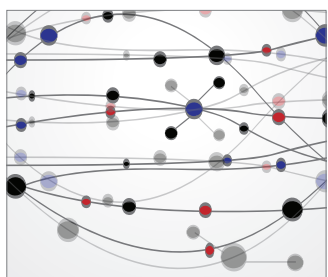

The Scientific World Journal
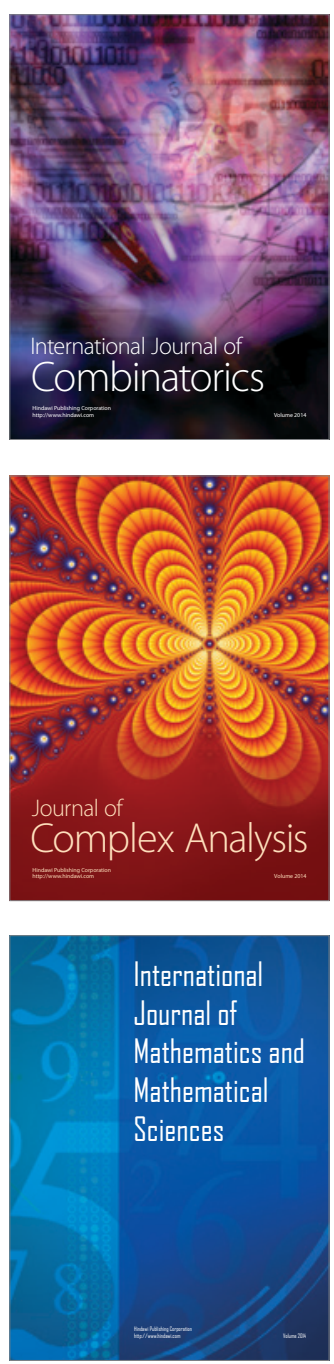
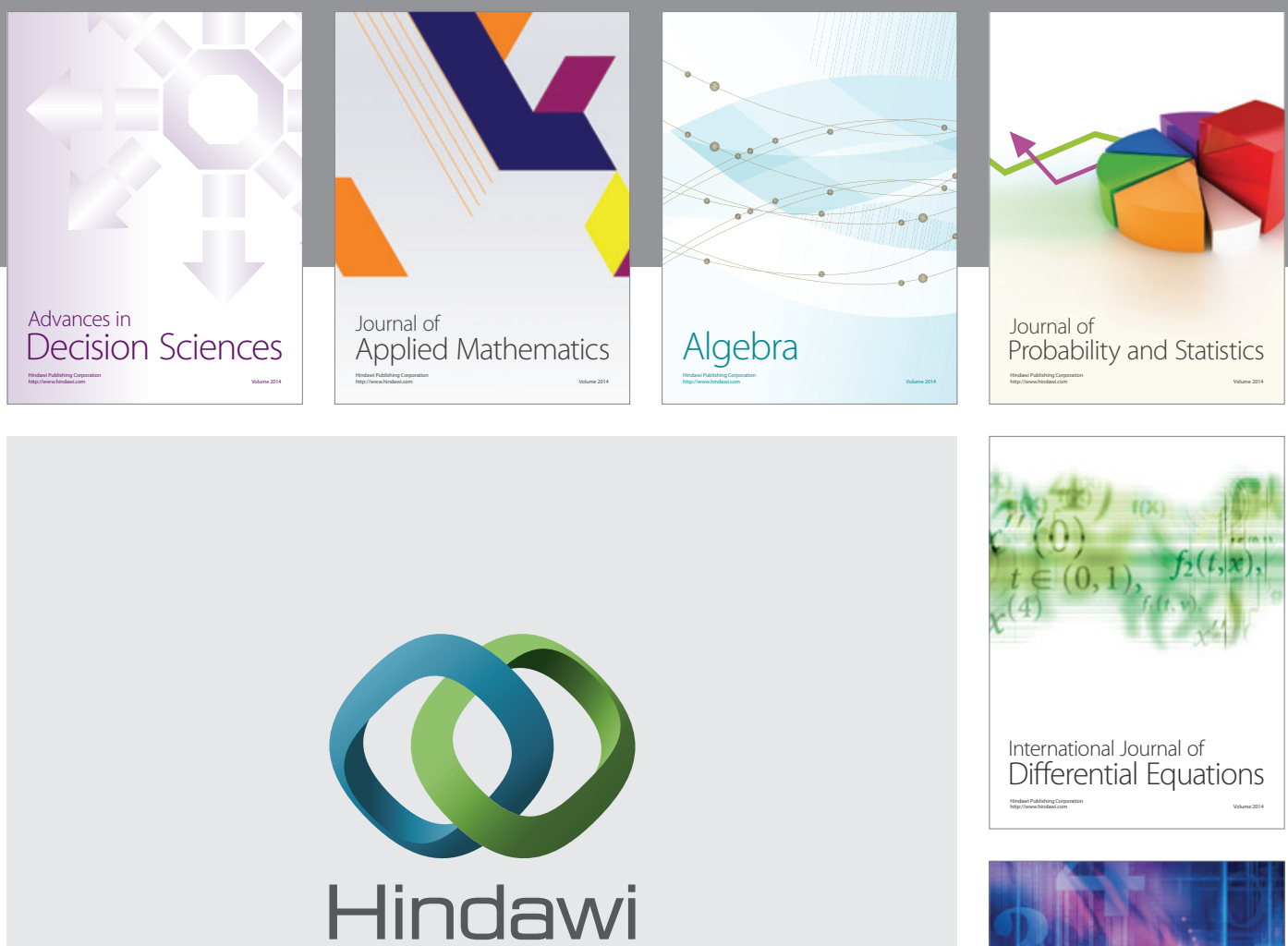

Submit your manuscripts at http://www.hindawi.com
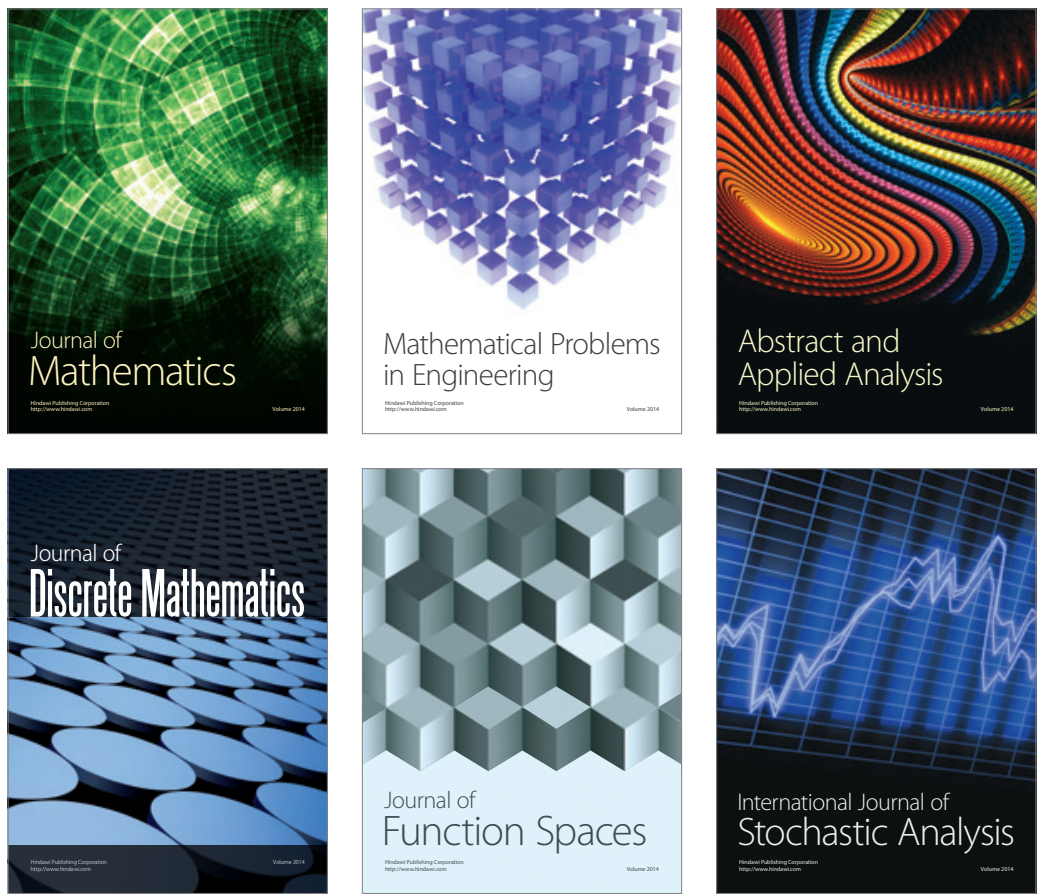

Journal of

Function Spaces

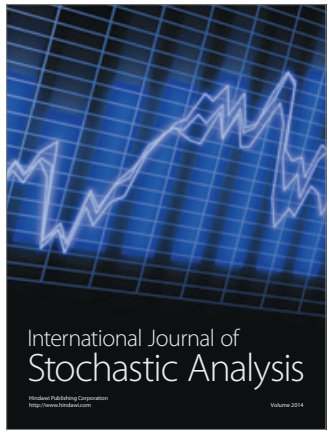

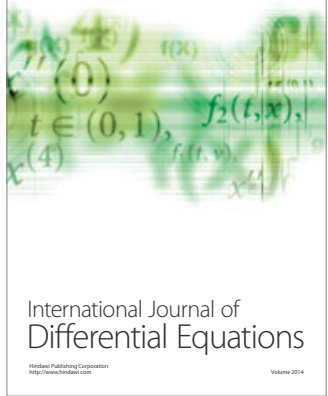
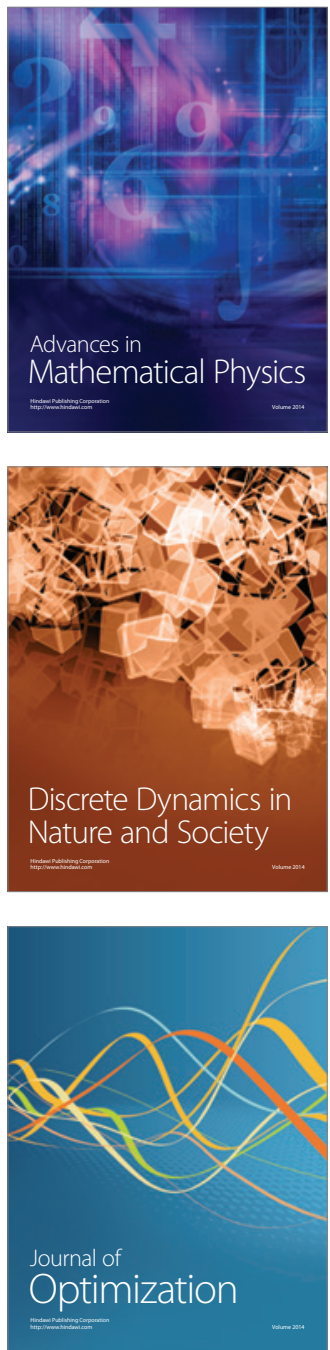